\title{
SPECT myocardial perfusion imaging as an adjunct to coronary calcium score for the detection of hemodynamically significant coronary artery stenosis
}

Franz von Ziegler ${ }^{1 *}$, Michaela Brendel ${ }^{1}$, Christopher Uebleis ${ }^{2}$, Susanne Helbig ${ }^{1}$, Martin Greif $^{1}$, Janine Ruemmler ${ }^{1}$, Christoph Becker ${ }^{3}$, Marcus Hacker ${ }^{2}$, Gerhard Steinbeck' and Alexander Becker ${ }^{1}$

\begin{abstract}
Background: Coronary artery calcifications (CAC) are markers of coronary atherosclerosis, but do not correlate well with stenosis severity. This study intended to evaluate clinical situations where a combined approach of coronary calcium scoring (CS) and nuclear stress test (SPECT-MPI) is useful for the detection of relevant CAD.

Methods: Patients with clinical indication for invasive coronary angiography (ICA) were included into our study during 08/2005-09/2008. At first all patients underwent CS procedure as part of the study protocol performed by either using a multidetector computed tomography $(C T)$ scanner or a dual-source $C T$ imager. CAC were automatically defined by dedicated software and the Agatston score was semi-automatically calculated. A stress-rest SPECT-MPI study was performed afterwards and scintigraphic images were evaluated quantitatively. Then all patients underwent ICA. Thereby significant CAD was defined as luminal stenosis $\geq 75 \%$ in quantitative coronary analysis (QCA) in $\geq 1$ epicardial vessel. To compare data lacking Gaussian distribution an unpaired Wilcoxon-Test (Mann-Whitney) was used. Otherwise a Students $t$-test for unpaired samples was applied. Calculations were considered to be significant at a $\mathrm{p}$-value of $<0.05$.
\end{abstract}

Results: We consecutively included 351 symptomatic patients (mean age: 61.2 \pm 12.3 years; range: 18-94 years; male: $\mathrm{n}=240$ ) with a mean Agatston score of 258.5 \pm 512.2 (range: $0-4214$ ). ICA verified exclusion of significant CAD in 66/67 (98.5\%) patients without CAC. CAC was detected in remaining 284 patients. In 132/284 patients (46.5\%) with CS $>0$ significant CAD was confirmed by ICA, and excluded in 152/284 (53.5\%) patients. Sensitivity for CAD detection by CS alone was calculated as $99.2 \%$, specificity was $30.3 \%$, and negative predictive value was $98.5 \%$. An additional SPECT in patients with CS>0 increased specificity to $80.9 \%$ while reducing sensitivity to $87.9 \%$. Diagnostic accuracy was $84.2 \%$.

Conclusions: In patients without $C S=0$ significant $C A D$ can be excluded with a high negative predictive value by CS alone. An additional SPECT-MPI in those patients with CS>0 leads to a high diagnostic accuracy for the detection of CAD while reducing the number of patients needing invasive diagnostic procedure.

Keywords: Computed tomography coronary calcium scoring, Single photon emission computed tomography myocardial perfusion imaging, Invasive coronary angiography, Coronary artery disease

\footnotetext{
* Correspondence: franz.ziegler@med.uni-muenchen.de

'University of Munich, Department of Cardiology, Campus Grosshadern,

Munich, Germany

Full list of author information is available at the end of the article
}

\section{Biomed Central}

(c) 2012 von Ziegler et al.; licensee BioMed Central Ltd. This is an Open Access article distributed under the terms of the Creative Commons Attribution License (http://creativecommons.org/licenses/by/2.0), which permits unrestricted use, distribution, and reproduction in any medium, provided the original work is properly cited. 


\section{Background}

Coronary artery calcifications (CAC) are a highly specific marker of coronary atherosclerosis, the extent of which is directly related to the atherosclerotic plaque burden [1]. According to a large number of clinical studies CAC is a reliable non-invasive diagnostic tool for the prediction of subsequent cardiovascular events in populations with elevated conventional risk factors [2-13]. In a large observational report 10 -year survival in asymptomatic patients with CAD was $99.4 \%$ in patients with a CAC score of 0 but was only $87.8 \%$ in those with a CAC score of greater than 1000 [14]. According to current guidelines in symptomatic patients calcium scoring (CS) might additionally serve as a useful filter prior to invasive coronary angiography or stress nuclear imaging due to its high sensitivity for flow-limiting CAD [15]. CAC studies including over 7,600 symptomatic patients demonstrate negative predictive values ranging from 96\% to $100 \%$, allowing for a high level of confidence that an individual in which CAC can be excluded has no obstructive disease $[11,12,16]$. However, specificity and diagnostic accuracy for prediction of flow-limiting stenosis in these patients is rather low [11-13]. Therefore, we hypothesize that combining the results of CS with the findings of a generally accepted functional imaging modality (such as single photon emission computed tomography [SPECT] or positron emission tomography [PET] myocardial perfusion imaging [MPI]) may enhance the prognostic value of both, resulting in benefits for patient screening and for the diagnosis and treatment of patients suspicious of having CAD [17-20]. Thus, the aim of our study was to evaluate different clinical scenarios in a research setting in which CS procedure alone or in combination with SPECT-MPI may enhance the diagnostic value of those non-invasive imaging modalities for the assessment of hemodynamically relevant $\mathrm{CAD}$ as detected by conventional invasive coronary angiography (ICA) in these particular patients.

\section{Methods}

\section{Patients}

An overall of 351 patients (mean age: $61.2 \pm 12.3$ years; range: 18-94 years; 240 male patients; 111 female patients) were consecutively included into our study during 08/2005-09/2008. All patients had a clinical indication for ICA due to either non conclusive stress test before (i.e. stress echocardiography or stress electrocardiogram) or typical stable angina with Class II angina pectoris symptoms at minimum according to the Canadian Cardiovascular Society Classification (CCSC) system [21]. Exclusion criteria were: (1) acute coronary syndrome including ST-elevation myocardial infarction, non-ST elevation myocardial infarction, or unstable angina; (2) positive Troponin in blood testing; (3) unstable clinical conditions; (4) known coronary artery disease after stent implantation procedure or coronary artery bypass surgery; (5) age < 18 years; (6) current pregnancy. The study was conducted at the University of Munich, Department of Cardiology, Campus Grosshadern, Munich, Germany and all patients gave written informed consent to participate. The study protocol was approved by the institutional review board and was in compliance with the Declaration of Helsinki. Table 1 gives a detailed overview of age and risk factors of all included patients. Patients were symptomatic with a mean CCSC of 2.4. Evaluation of cardiovascular risk profile revealed a moderate to high pre-test probability of CAD with an average number of 2.4 conventional cardiovascular risk factors.

\section{Coronary calcium scoring}

CS procedure was performed by either using a multidetector CT scanner (Sensation 64; Siemens; Forchheim; Germany) or a dual-source CT imager (Definition; Siemens; Forchheim; Germany) in the thin-section mode. Standardized acquisition protocol for both scanner types included a tube voltage of $120 \mathrm{kV}$ at a current of $80 \mathrm{mAs}$. Rotation time was $0.33 \mathrm{~s}$. Detector collimation was $64 \times 0.6 \mathrm{~mm}$. Acquired images were reconstructed using a B35f Kernel, reconstruction increment was $1.5 \mathrm{~mm}$ resulting in a $50 \%$ overlap. Electrocardiographically triggered images were acquired at $80 \%$ of the R-R interval during one end-inspiratory breath-hold period. All images were electronically transferred to one dedicated workstation for CS evaluation (Leonardo; Siemens; Forchheim; Germany). Thereby, CAC were automatically defined as lesions with attenuations greater than 130 Hounsfield Units in more than four adjacent pixels. For quantification of CAC, the Agatston score was calculated as the product of the lesion's surface area and a weighting factor ranging from

Table 1 Baseline characteristics of all $\mathbf{3 5 1}$ individuals included in the study

\begin{tabular}{llll}
\hline & All patients & Male patients & Female patients \\
\hline & $351(100.0 \%)$ & $240(68.4 \%)$ & $111(31.6 \%)$ \\
Mean age (years) & $61.2 \pm 12.3$ & $60.0 \pm 12.2$ & $63.7 \pm 12.2$ \\
Hypertension & $236(67.2 \%)$ & $150(62.5 \%)$ & $86(77.5 \%)$ \\
Family history & $118(33.6 \%)$ & $85(35.4 \%)$ & $33(29.7 \%)$ \\
Smoking & $115(32.8 \%)$ & $88(36.7 \%)$ & $27(18.9 \%)$ \\
Hyperlipidemia & $196(55.8 \%)$ & $144(60.0 \%)$ & $52(46.9 \%)$ \\
Adipositas & $26(7.4 \%)$ & $13(5.4 \%)$ & $13(11.7 \%)$ \\
Diabetes & $38(10.8 \%)$ & $22(9.2 \%)$ & $16(14.4 \%)$ \\
Average no. of & $2.4 \pm 0.9$ & $2.5 \pm 1.1$ & $2.3 \pm 1.0$ \\
risk factors & & & \\
Mean CCS Score & $2.4 \pm 0.9$ & $2.4 \pm 0.7$ & $2.4 \pm 0.8$ \\
\hline
\end{tabular}


one to four, which was assigned according to the peak attenuation of the lesion [22]. All CS scans were evaluated by a physician unaware of the patients' clinical diagnoses.

\section{SPECT Myocardial Perfusion Imaging}

A 1-day technetium $99 \mathrm{~m}$ methoxyisobutyl-isonitrile (Cardiolite; Bristol-Myers Squibb Medical Imaging; North Billerica; Massachusetts) stress-rest SPECT MPI protocol was used for all patients, as described previously [23]. Whenever possible patients were stressed ergometrically on a stationary bicycle; otherwise a pharmacological stress examination protocol was used. Thereby bodyweight- adjusted $0.56 \mathrm{mg}$ dipyramidole $/ \mathrm{kg}$ for an overall of 4 minutes was administered. No attenuation correction or gated SPECT acquisition was performed. Scintigraphic stress and rest images were evaluated quantitatively by using a dedicated software (AutoQUANT 6.0; Philips Medical Systems; Cleveland; Ohio) [24-26] that employs a 20-segment left ventricular model with a common five-point scoring system $(0=$ "normal perfusion"; 5= "no perfusion") [22,27]. Results were interpreted by two investigators blinded to CS and ICA results. The summed stress scores (SSS) and summed rest scores (SRS) of all segments were determined. The summed difference score (SDS) was calculated as the difference between the SSS and the SRS. A SDS $\geq 4$ was defined as stress-induced ischemia and counted as pathological SPECT-MPI.

\section{Conventional invasive coronary angiography}

ICA was performed by the Judkins approach according to a standardized protocol using a 6-F catheter. Examinations were blinded to the results of CS as well as SPECT-MPI. Significant CAD was defined as luminal stenosis $\geq 75 \%$ in quantitative coronary analysis (QCA) in $\geq 1$ epicardial vessel. For QCA the Quantcor QCA software package (Quantcor QCA; CAAS II; V 5.0; Pie Medical Imaging; Maastricht; Netherlands) was used. The decision for coronary intervention (either stent implantation procedure or coronary artery bypass surgery) was independently made by the examiner, who was blinded to the CS and SPECT-MPI results.

\section{Statistical analysis}

All statistical analyses were performed using the SigmaStat software package (SigmaStat for Windows; V 3.10; Systat Software GmbH; Erkrath; Germany). To compare data lacking Gaussian distribution an unpaired Wilcoxon-Test (Mann-Whitney) was used. Otherwise a Students $t$-test for unpaired samples was applied. To compare the diagnostic power of CS alone or in combination with SPECT-MPI a ROC analysis was performed. All numbers are given in mean \pm standard deviation. Calculations were considered to be significant at a p-value of $<0.05$.

\section{Results}

\section{Coronary calcium scoring}

CS procedure was performed without any complication in all patients with diagnostic image quality. Thus, none of the patients had to be excluded from further analysis. Mean coronary calcium Agatston score was 258.5 \pm 512.2 (range: $0-4213.7$ ). In an overall of 67 patients (38 male; 29 female) coronary calcifications were excluded. In the remaining 284 patients (202 male; 82 female) calcified tissue was detected. Patients with CAC (mean age: $63.1 \pm 10.9$ years; range: $31-94$ years) were significantly older than patients without calcified tissue (53.0 \pm 14.3 years; range: $18-80$ years; $\mathrm{p}<0.001)$. As expected, the Agatston score increased with elevated patient age. Additionally, female patients showed lower overall CS values.

\section{SPECT Myocardial Perfusion Imaging}

All stress-rest SPECT-MPI examinations were performed without complications. 230 (65.4\%) patients were stressed ergometrically on a stationary bicycle; and 121 (34.5\%) patients, pharmacologically. In 303 (86.3\%) patients, $0.8 \mathrm{mg}$ of nitroglycerine was administered sublingually before injection of the radiopharmaceutical for the rest image.

A stress induced myocardial ischemia was detected in 148 patients (42.2\%; 117 male; 31 female; mean age: $65.0 \pm 10.6$ years; range: $35-94$ years). In remaining 203 patients (57.8\%; 123 male; 80 female) being significantly younger (mean age: $58.4 \pm 12.7$ years; range: 18-85 years; $\mathrm{p}<0.001)$ stress induced ischemia was excluded.

\section{Coronary artery disease detection}

All invasive examinations were performed without any complication. An overall of 133 patients (37.9\%; mean age $65.3 \pm 10.1$ years; range: $37-94$ years) had significant CAD in ICA. This population consisted of 108 male (mean age: 64.2 \pm 9.2 years; range: $43-94$ years) and 25 female patients (mean age: $69.9 \pm 12.4$ years; range: 37-86 years). A coronary 1-vessel disease was found in 64 cases $(48.1 \%), 35$ patients $(26.3 \%)$ had a 2 -vessel disease, and all 3 coronary vessels were significantly diseased in 34 patients (25.6\%). Stent implantation procedure was performed in 94 patients $(70.7 \%)$ and remaining 39 patients (29.3\%) underwent bypass surgery. Significant CAD was excluded by ICA in remaining 218 patients (62.1\%; mean age: $58.7 \pm 12.8$ years; range: $18-87$ years) being significantly younger than patients with hemodynamically relevant CAD $(\mathrm{p}<0.001)$. 
In 66/67 (98.5\%) patients without coronary calcifications significant CAD leading to intervention was excluded by ICA. Only one 65-year old female patient had coronary 1-vessel disease leading to intervention in left anterior coronary artery stenosis (drug-eluting stent implantation procedure). 132/284 patients (46.5\%) with positive CS also showed significant disease in ICA. Remaining 152 patients with detectable calcified tissue had no significant CAD. Thus, we calculated a sensitivity of $99.2 \%$, a specificity of $30.3 \%$, a negative predictive value (NPV) of $98.5 \%$, and a positive predictive value (PPV) of $46.5 \%$ for calcium scoring alone with respect to significant CAD detection. Diagnostic accuracy (DA) was $56.4 \%$. Table 2 gives a detailed overview over CS results using different thresholds.

In an overall of $117 / 148$ patients $(79.1 \%)$ with myocardial ischemia in SPECT-MPI (mean age: $65.0 \pm 10.6$ years; range: $35-95$ years) significant CAD was confirmed by ICA. False-positive results of SPECT-MPI were found in remaining 31/148 patients (21.0\%). In an overall of $187 /$ 203 patients (92.2\%) SPECT correctly excluded significant CAD. False-negative results were found in remaining 16/203 patients (7.9\%). Thus, using SPECT imaging as stand alone modality for significant CAD detection revealed a sensitivity of $88 \%$, a specificity of $85.8 \%$, a NPV of $92.1 \%$, and a PPV of $79.1 \%$. DA for SPECT-MPI was $86.6 \%$. ROC analysis of SPECT-MPI alone vs. SPECT-MPI in combination with CS revealed no significant difference; 0.869 (95\%-CI 0.829-0.902) vs. 0.870 (95\%-CI 0.830-0.903) ( $\mathrm{p}=0.902)$. Mean CS in patients with ischemic myocardium in SPECT was significantly higher than in patients without inducible ischemia (518.6 \pm 676.4 vs. $69.4 \pm 192.4 ; \mathrm{p}<0.05)$.

In patients with calcified plaque tissue SPECT-MPI detected stress induced ischemia in 145/284 patients (51.1\%). Of these patients, 116 (80.0\%) suffered from significant CAD as confirmed by ICA. In remaining 29 patients ICA excluded significant disease. In patients without inducible ischemia in SPECT-MPI but with positive CS $(n=139)$ ICA confirmed exclusion of significant $\mathrm{CAD}$ in $123 / 139$ (88.5\%). False negative results were found in 16 patients $(11.5 \%)$. Latter patients were significantly older compared to the general patients without detectable ischemia in our study population

Table 2 Diagnostic performance of CS using different thresholds compared to SPECT-MPI

\begin{tabular}{llllll}
\hline & Sensitivity & Specificity & PPV & NPV & DA \\
\hline CS $=0$ & $99.2 \%$ & $30.3 \%$ & $46.5 \%$ & $98.5 \%$ & $56.4 \%$ \\
CS $<10$ & $93.2 \%$ & $52.8 \%$ & $54.6 \%$ & $92.7 \%$ & $68.1 \%$ \\
CS $<100$ & $81.2 \%$ & $83.5 \%$ & $75.0 \%$ & $87.9 \%$ & $82.6 \%$ \\
CS $<400$ & $40.6 \%$ & $95.4 \%$ & $84.4 \%$ & $72.5 \%$ & $74.6 \%$ \\
SPECT-MPI & $88.0 \%$ & $85.8 \%$ & $79.1 \%$ & $92.1 \%$ & $86.6 \%$ \\
\hline
\end{tabular}

(mean age: $67.3 \pm 9.3$ years vs. $58.4 \pm 12.7$ years; $\mathrm{p}=0.0033$ ), and additionally showed a significantly higher overall amount of calcified tissue (mean CS: $303.1 \pm 320.6$ vs. 69.4 $\pm 192.4 ; \mathrm{p}<0.0001)$.

If coronary calcium scoring would have been used as a kind of filter before SPECT imaging, disease prevalence in this subgroup of patients with positive calcium scores would increase to $46.5 \%(132 / 284)$. Utilizing this approach a sensitivity of $87.9 \%$ with a specificity of $80.9 \%$ would have been calculated, and thus, NPV would be 88.5\%, and PPV 80.0\%. Theoretically, in 67 patients further scanning procedure would have been avoided, with one false negative result in aforementioned female patient. Adding additional 139 patients with positive CS, but negative SPECT-MPI studies an overall of 206/351 (58.7\%) patients would have been prevented from invasive procedure with an overall of 17 (5.8\%) false-negative results.

\section{Discussion}

According to currently published reports only roughly one third of all heart catheterizations performed are leading to intervention [28]. To improve diagnostic yield the current guidelines of international cardiology societies suggest non-invasive tests for the detection of CAD in symptomatic patients, such as stress echocardiography with reported pooled sensitivity of $80-85 \%$, and specificity of $85-86 \%$, before invasive procedure [29]. Calcium scoring is a non-invasive modality which detects coronary calcifications being a surrogate marker for cardiac atherosclerosis. According to current literature the amount of calcified tissue correlates with the risk of developing adverse cardiac events in the future, such as myocardial infarction or sudden cardiac death. In addition it seems that CS is a better risk predictor for prospective cardiac events than currently available risk stratification score systems, such as Framingham or ATPIII risk score, in the way that CS reflects individual atherosclerotic disease burden. Especially the exclusion of coronary calcifications has been shown to exclude significant CAD in symptomatic patients [11-13]. However, the amount of calcium does not correlate well with stenosis severity [30]. Thus, aim of our study was to evaluate the use of an approach combining CS determination and SPECT-MPI. Especially we intended to identify the settings in which one can refrain from additional imaging or incremental information is won by the combination of both modalities.

In our patient population of 351 consecutive patients admitted for invasive coronary angiography, disease prevalence of significant CAD needing intervention was $38 \%$ reflecting a typical population undergoing ICA. As expected, specificity and positive predictive value of coronary calcium scoring were rather low. Thus, detection 
of hemodynamically relevant stenosis of epicardial vessels utilizing this modality is not practicable. However, a high sensitivity of $99.2 \%$, and a high NPV of $98.5 \%$ suggest CS being a useful tool for ruling out CAD by exclusion of calcified plaque tissue. In these patients an additional SPECT-MPI did not further increase diagnostic accuracy as the negative predictive value of SPECTMPI is limited to $92.1 \%$. Following these results we would have correctly refrained from ICA and SPECTMPI in 66 patients $(18.8 \%)$ of our study population. Although there are some publications questioning the safety of a zero to low coronary calcium scores for the purpose of CAD exclusion in symptomatic patients, several studies in large patient cohorts consistently have shown a high NPV of $>95 \%$ utilizing a threshold of zero calcium similar to our study. But also in these studies the safety of ruling out CAD is reduced by using higher thresholds like a CS of 10 or a CS above the 25th age and sex adjusted percentile instead of the exclusion of calcified tissue [11-13]. The same was true for our study: increasing CS thresholds would lead to an increase in specificity and PPV. Concomitantly sensitivity and NPV decreased to a value of $40.6 \%$ and $72.5 \%$, respectively. Thus CS would no longer be suitable as a filter for invasive angiography and still not sufficient for the detection of relevant stenosis with a PPV of $84.4 \%$. Still, diagnostic accuracy of CS at a threshold of 100 is comparable to the diagnostic performance of SPECT-MPI.

Due to low specificities of positive coronary calcium scores, a functional test like SPECT-MPI following initial CS in case of calcified tissue detection is reasonable. In our 284 patients with detectable coronary calcifications $51.1 \%(n=145)$ showed ischemia in SPECT-MPI followed by intervention in $80.0 \%(n=116)$ of those patients. Specificity of this combined approach increased to $81 \%$ with a disease prevalence of now $46.5 \%$. Additionally a positive ischemic burden and its relation to a specific coronary vessel territory, provides helpful information for guiding interventional procedure.

In remaining 139 patients with positive calcium scores, SPECT-MPI excluded significant CAD in 123 patients (88.5\%). Although CS did not help further with respect to CAD detection in these particular patients, it nonetheless provides individual prognostic information and estimates risk of developing prospective major coronary events. In that respect CS might outperform SPECTMPI in symptomatic patients in the long-time follow-up [31]. An overall of 16 patients (11.5\%) were misclassified by SPECT-MPI. Mean CS of these patients exceeded 300 , with below 100 in only three cases, so that the majority of these patients presumably would have been put on secondary prevention therapy due to the CS results [32]. Furthermore it remains questionable whether these patients in particular would benefit from additional revascularization according to the nuclear medicine substudy of the Clinical Outcomes Utilizing Revascularization and Aggressive Drug Evaluation (COURAGE) trial [33]. In this study Shaw et al. compared the effectiveness of adding coronary revascularization in order to minimize stress-induced ischemia with that of optimal medical therapy, utilizing SPECT-MPI to find the end point. They found that the outcome in patients with stable CAD was most strongly associated with the amount of residual ischemia after medical, or revascularization therapy, or both.

\section{Limitations}

The utilization of different scanner technologies (MDCT and DSCT scanners) poses the risk of inconsistent calcium score values. According to a currently published head-to-head comparison of these two scanner types by Ghadri et al. revealed an excellent inter-scanner agreement for Agatston scores with a correlation coefficient of 0.976 despite relatively wide limits of agreement and a coefficient of variation $15.1 \%$ even in case of different vendors [34]. Calcium mass score as well as calcium volume score were also evaluated showing also excellent correlation, but wider limits of agreement in BlandAltman Analysis. Interestingly the authors found that the use of different workstations had a greater influence on the comparability than the scanner technology. To account for those possible sources of error a comparable acquisition protocol for both scanner technologies routinely is used at our institution and the same workstation with the same software solution was utilized to calculate the Agatston score. Indication for revascularization was solely based on detection of significant stenosis $(\geq 75 \%)$ in QCA and was independently made by the examiner without necessary verification of existing ischemia in order to guarantee for a blinded study design. This approach to revascularization procedure represents clinical practice at the time of study initiation. For comparison of SPECT-MPI a functional invasive measurement such as fractional flow reserve (FFR) would have been more appropriate, but FFR usually is not a widely available diagnostic modality. The use of the $\geq 75 \%$ stenosis-degree criterion in order to define hemodynamically relevant CAD theoretically results in a higher number of false-positive findings with initially lower specificity and PPV. The likelihood of a $\geq 50 \%$-stenosis causing ischemia, which serves as CAD defining endpoint in the majority of studies is relatively low and does not warrant revascularization procedure in the majority of cases unless prove of ischemic burden. Additionally selection bias must be considered while interpreting the data presented. First our evaluation was based on patients with clinical indication for ICA as referred by primary-care providers that do not necessarily sent unselected chest-pain patients for 
cardiac evaluation. Second our study was conducted at a single university site including only regional patients. Thus our study population may not reflect general symptomatic CAD patients.

\section{Conclusions}

It was the aim of the study to evaluate the diagnostic accuracy of a combined approach using CS and SPECTMPI. In patients with a $\mathrm{CS}=0$ an additional SPECT-MPI did not lead to an increased diagnostic accuracy. The exclusion of CAD was attained with a high NPV by CS alone. In patients with a positive CS, SPECT-MPI increased specificity and PPV for the detection of CAD significantly.

In patients with a regular SPECT-MPI CS gave additional information on patients' coronary atherosclerosis and cardiovascular risk.

\section{Abbreviations \\ CAC: coronary artery calcifications; CAD: coronary artery disease; CS: calcium scoring; CT: computed tomography; DA: diagnostic accuracy; ICA: invasive coronary angiography; MPI: myocardial perfusion imaging; NPV: negative predictive value; PET: positron emission tomography; PPV: positive predictive value; QCA: quantitative coronary analysis; SDS: summed difference score; SPECT: single photon emission computed tomography; SRS: summed rest scores; SSS: summed stress scores.}

\section{Competing interests}

The authors declare that they have no competing interests.

\section{Authors' contributions}

FVZ is responsible for coordination of the study, statistical evaluation and manuscript preparation. $\mathrm{MB}$ is responsible for data acquisition and statistical evaluation. CU and MH are responsible for SPECT-MPI data acquisition and evaluation. $\mathrm{SH}$ is responsible for data acquisition and data base maintenance. $M G$ and JR are responsible for ICA studies. CB is responsible for $C T$ scanning and CS evaluation. GS supervised ICA performance and interpretation. $A B$ contributed in conception and design of the study and is responsible for manuscript review. All authors have read and approved the final manuscript.

\section{Author details}

${ }^{1}$ University of Munich, Department of Cardiology, Campus Grosshadern, Munich, Germany. ${ }^{2}$ University of Munich, Department of Nuclear Medicine, Campus Grosshadern, Munich, Germany. ${ }^{3}$ University of Munich, Department of Clinical Radiology, Campus Grosshadern, Munich, Germany.

Received: 10 May 2012 Accepted: 30 November 2012

Published: 4 December 2012

\section{References}

1. Rumberger JA, Simons DB, Fitzpatrick LA, Sheedy PF, Schwartz RS: Coronary artery calcium area by electron-beam computed tomography and coronary atherosclerotic plaque area. A histopathologic correlative study. Circulation 1995, 92(8):2157-2162.

2. Arad Y, Goodman KJ, Roth M, Newstein D, Guerci AD: Coronary calcification, coronary disease risk factors, C-reactive protein, and atherosclerotic cardiovascular disease events: the St. Francis Heart Study. J Am Coll Cardiol 2005, 46(1):158-165.

3. Detrano R, Guerci AD, Carr JJ, Bild DE, Burke G, Folsom AR, Liu K, Shea S, Szklo M, Bluemke DA, et al: Coronary calcium as a predictor of coronary events in four racial or ethnic groups. N Engl J Med 2008 358(13):1336-1345.

4. Kondos GT, Hoff JA, Sevrukov A, Daviglus ML, Garside DB, Devries SS, Chomka EV, Liu K: Electron-beam tomography coronary artery calcium and cardiac events: a 37-month follow-up of 5635 initially asymptomatic low- to intermediate-risk adults. Circulation 2003, 107(20):2571-2576.
5. Raggi P, Callister TQ, Cooil B, He ZX, Lippolis NJ, Russo DJ, Zelinger A, Mahmarian JJ: Identification of patients at increased risk of first unheralded acute myocardial infarction by electron-beam computed tomography. Circulation 2000, 101(8):850-855.

6. Shaw LJ, Raggi P, Schisterman E, Berman DS, Callister TQ: Prognostic value of cardiac risk factors and coronary artery calcium screening for all-cause mortality. Radiology 2003, 228(3):826-833.

7. Taylor AJ, Bindeman J, Feuerstein I, Cao F, Brazaitis M, O'Malley PG: Coronary calcium independently predicts incident premature coronary heart disease over measured cardiovascular risk factors: mean three-year outcomes in the Prospective Army Coronary Calcium (PACC) project. J Am Coll Cardiol 2005, 46(5):807-814.

8. Wayhs R, Zelinger A, Raggi P: High coronary artery calcium scores pose an extremely elevated risk for hard events. J Am Coll Cardiol 2002, 39(2):225-230.

9. Wong ND, Hsu JC, Detrano RC, Diamond G, Eisenberg H, Gardin JM: Coronary artery calcium evaluation by electron beam computed tomography and its relation to new cardiovascular events. Am J Cardiol 2000, 86(5):495-498

10. Becker A, Leber A, Becker C, Knez A: Predictive value of coronary calcifications for future cardiac events in asymptomatic individuals. Am Heart J 2008, 155(1):154-160.

11. Knez A, Becker A, Leber A, White C, Becker CR, Reiser MF, Steinbeck G, Boekstegers $P$ : Relation of coronary calcium scores by electron beam tomography to obstructive disease in 2,115 symptomatic patients. Am J Cardiol 2004, 93(9):1150-1152.

12. Haberl R, Becker A, Leber A, Knez A, Becker C, Lang C, Bruning R, Reiser M, Steinbeck G: Correlation of coronary calcification and angiographically documented stenoses in patients with suspected coronary artery disease: results of 1,764 patients. J Am Coll Cardiol 2001, 37(2):451-457

13. Sarwar A, Shaw $L$, Shapiro MD, Blankstein R, Hoffmann U, Cury RC, Abbara S, Brady TJ, Budoff MJ, Blumenthal RS, et al: Diagnostic and prognostic value of absence of coronary artery calcification. JACC Cardiovasc Imaging 2009, 2(6):675-688

14. Budoff MJ, Shaw $\sqcup$, Liu ST, Weinstein SR, Mosler TP, Tseng PH, Flores FR, Callister TQ, Raggi P, Berman DS: Long-term prognosis associated with coronary calcification: observations from a registry of 25,253 patients. J Am Coll Cardiol 2007, 49(18):1860-1870.

15. Greenland $\mathrm{P}$, Bonow RO, Brundage BH, Budoff MJ, Eisenberg MJ, Grundy SM, Lauer MS, Post WS, Raggi P, Redberg RF, et al: ACCF/AHA 2007 clinical expert consensus document on coronary artery calcium scoring by computed tomography in global cardiovascular risk assessment and in evaluation of patients with chest pain: a report of the American College of Cardiology Foundation Clinical Expert Consensus Task Force (ACCF/AHA Writing Committee to Update the 2000 Expert Consensus Document on Electron Beam Computed Tomography). Circulation 2007, 115(3):402-426.

16. Budoff MJ, Diamond GA, Raggi P, Arad Y, Guerci AD, Callister TQ, Berman D: Continuous probabilistic prediction of angiographically significant coronary artery disease using electron beam tomography. Circulation 2002, 105(15):1791-1796

17. Gibbons RJ, Abrams J, Chatterjee K, Daley J, Deedwania PC, Douglas JS, Ferguson TB Jr, Finn SD, Fraker TD Jr, Gardin JM, et al: ACC/AHA 2002 guideline update for the management of patients with chronic stable angina-summary article: a report of the American College of Cardiology/ American Heart Association Task Force on Practice Guidelines (Committee on the Management of Patients With Chronic Stable Angina). Circulation 2003, 107(1):149-158.

18. Ramakrishna G, Miller TD, Breen JF, Araoz PA, Hodge DO, Gibbons RJ: Relationship and prognostic value of coronary artery calcification by electron beam computed tomography to stress-induced ischemia by single photon emission computed tomography. Am Heart J 2007, 153(5):807-814.

19. Rozanski A, Gransar H, Wong ND, Shaw $\amalg$, Miranda-Peats R, Polk D, Hayes SW, Friedman JD, Berman DS: Clinical outcomes after both coronary calcium scanning and exercise myocardial perfusion scintigraphy. J Am Coll Cardiol 2007, 49(12):1352-1361.

20. Schenker MP, Dorbala S, Hong EC, Rybicki FJ, Hachamovitch R, Kwong RY, Di Carli MF: Interrelation of coronary calcification, myocardial ischemia, and outcomes in patients with intermediate likelihood of coronary 
artery disease: a combined positron emission tomography/computed tomography study. Circulation 2008, 117(13):1693-1700.

21. Campeau L: Letter: grading of angina pectoris. Circulation 1976, 54(3):522-523.

22. Agatston AS, Janowitz WR, Hildner FJ, Zusmer NR, Viamonte M Jr, Detrano R: Quantification of coronary artery calcium using ultrafast computed tomography. J Am Coll Cardiol 1990, 15(4):827-832.

23. Hacker M, Jakobs T, Matthiesen F, Vollmar C, Nikolaou K, Becker C, Knez A, Pfluger T, Reiser M, Hahn K, et al: Comparison of spiral multidetector CT angiography and myocardial perfusion imaging in the noninvasive detection of functionally relevant coronary artery lesions: first clinical experiences. J Nucl Med 2005, 46(8):1294-1300.

24. Germano G, Kavanagh PB, Berman DS: An automatic approach to the analysis, quantitation and review of perfusion and function from myocardial perfusion SPECT images. Int I Card Imaging 1997, 13(4):337-346.

25. Germano G, Kavanagh PB, Waechter P, Areeda J, Van Kriekinge S, Sharir T, Lewin HC, Berman DS: A new algorithm for the quantitation of myocardial perfusion SPECT. I: technical principles and reproducibility. J Nucl Med 2000, 41(4):712-719.

26. Slomka PJ, Nishina H, Berman DS, Kang X, Friedman JD, Hayes SW, Aladl UE, Germano G: Automatic quantification of myocardial perfusion stress-rest change: a new measure of ischemia. J Nucl Med 2004, 45(2):183-191.

27. Hachamovitch R, Berman DS, Shaw LJ, Kiat H, Cohen I, Cabico JA, Friedman J, Diamond GA: Incremental prognostic value of myocardial perfusion single photon emission computed tomography for the prediction of cardiac death: differential stratification for risk of cardiac death and myocardial infarction. Circulation 1998, 97(6):535-543.

28. Cook S, Togni M, Walpoth N, Maier W, Muehlberger V, Legrand V, Milicic D, Zambartas C, Zelizko M, Madsen JK, et al: Percutaneous coronary interventions in Europe 1992-2003. Eurolntervention 2006, 1(4):374-379.

29. Fox K, Garcia MA, Ardissino D, Buszman P, Camici PG, Crea F, Daly C, De Backer G, Hjemdahl P, Lopez-Sendon J, et al: Guidelines on the management of stable angina pectoris: executive summary: the Task Force on the Management of Stable Angina Pectoris of the European Society of Cardiology. Eur Heart J 2006, 27(11):1341-1381.

30. Schmermund A, Mohlenkamp S, Mathes P, Erbel R: [Value of coronary artery calcium measurements in primary prevention]. Z Kardiol 2005, 94(3):79-87.

31. Uebleis C, Becker A, Griesshammer I, Cumming P, Becker C, Schmidt M, Bartenstein P, Hacker M: Stable coronary artery disease: prognostic value of myocardial perfusion SPECT in relation to coronary calcium scoring-long-term follow-up. Radiology 2009, 252(3):682-690.

32. Mohlenkamp S, Lehmann N, Greenland P, Moebus S, Kalsch H, Schmermund A, Dragano N, Stang A, Siegrist J, Mann K, et al: Coronary artery calcium score improves cardiovascular risk prediction in persons without indication for statin therapy. Atherosclerosis 2011, 215(1):229-236

33. Shaw LJ, Berman DS, Maron DJ, Mancini GB, Hayes SW, Hartigan PM, Weintraub WS, O'Rourke RA, Dada M, Spertus JA, et al: Optimal medical therapy with or without percutaneous coronary intervention to reduce ischemic burden: results from the Clinical Outcomes Utilizing Revascularization and Aggressive Drug Evaluation (COURAGE) trial nuclear substudy. Circulation 2008, 117(10):1283-1291.

34. Ghadri JR, Goetti R, Fiechter M, Pazhenkottil AP, Kuest SM, Nkoulou RN, Windler C, Buechel RR, Herzog BA, Gaemperli O, et al: Inter-scan variability of coronary artery calcium scoring assessed on 64-multidetector computed tomography vs. dual-source computed tomography: a head-to-head comparison. Eur Heart J 2011, 32(15):1865-1874.

doi:10.1186/1471-2261-12-116

Cite this article as: von Ziegler et al:: SPECT myocardial perfusion imaging as an adjunct to coronary calcium score for the detection of hemodynamically significant coronary artery stenosis. BMC

Cardiovascular Disorders 2012 12:116.

\section{Submit your next manuscript to BioMed Central and take full advantage of:}

- Convenient online submission

- Thorough peer review

- No space constraints or color figure charges

- Immediate publication on acceptance

- Inclusion in PubMed, CAS, Scopus and Google Scholar

- Research which is freely available for redistribution

Submit your manuscript at www.biomedcentral.com/submit
Ciomed Central 